\title{
MiR-200a promotes the survival of cardiac cells and improves cardiac injury in chronic heart failure rats
}

\author{
Guiping $\mathrm{Wu}^{1}$, Xiaowen $\mathrm{Che}^{2}$
}

${ }^{1}$ Department of Cardiology, Shanxi Provincial People's Hospital, Taiyuan, Shanxi, China

${ }^{2}$ Department of AIDS Prevention and Control, Taiyuan Center for Disease Control and Prevention, Taiyuan, Shanxi, China

Submitted: 22 February 2020

Accepted: 12 March 2020

Arch Med Sci

DOI: https://doi.org/10.5114/aoms.2020.94500

Copyright $\odot 2020$ Termedia \& Banach

\section{Abstract}

Introduction: miRNAs play an important role in cardiovascular abnormalities such as heart failure. In the present work we evaluated the role of miR-200a in the condition of chronic heart failure and also the mechanism involved.

Material and methods: In the study 180 subjects, among whom 100 were reported for chronic heart failure and 80 as normal, were included. ELISA and qRT-PCR was done to evaluate levels of HMGB1 and miR-200a in subjects. The cardiac hemodynamics and functioning, oxidative stress and expression of mediators of inflammation were studied in rats with chronic heart failure induced after transfecting them with miR-200a or HMGB1. Luciferase activity was measured to establish any correlation between HMGB1 and miR-200a.

Results: The chronic heart failure patients included in the study showed suppressed levels of miR-200a and elevated HMGB1 compared to normal subjects. In chronic heart failure rats, the transfection of miR-200a attenuated the cardiac function and other hemodynamic parameters. In addition, improvement in oxidative stress as well as inflammatory mediators was observed. The outcomes also confirmed that HMGB1 was the potential target of miR-200a. It was also noted that upon transfection miR-200a resulted in suppression of protein as well as mRNA levels of HMGB1 in the cardiac tissue of chronic heart failure rats. Also overexpression of HMGB1 decreased the effects of miR-200a.

Conclusions: The outcomes indicate that miR-200a exerts a protective effect on cardiac cell injury via the HMGB1 pathway.

Key words: HMGB1, chronic heart failure, miR-200a.

\section{Introduction}

Heart failure (HF) is a condition with abnormal cardiac function or heart structure which leads to inadequacy of oxygen required for normal metabolic reactions in tissues [1, 2]. Chronic heart failure (CHF) has been responsible for deaths worldwide with the progression of HF [3]. Even though the research in treating $\mathrm{HF}$ has advanced in recent times, the mortality rate in patients remains high. Prevalence of diabetes and increasing age have led to an increased rate of HF globally [4]. There is an urgent need for novel therapies for treating HF.

MicroRNAs (miRs) are small non-coding RNAs measuring 18-25 nt in length and are found to be potential gene regulators required for differentiation, proliferation and survival of cells [5]. Hence, miRs are regarded

\author{
Corresponding author: \\ Dr. Guiping Wu \\ Department of Cardiology \\ Shanxi Provincial \\ People's Hospital \\ 29 Shuangtasi St \\ Taiyuan 030000 \\ Shanxi, China \\ Phone/fax: +86351 4960130 \\ E-mail: YeddaHeddayNyLel@ \\ yahoo.com
}


to play an important role in major physiological and pathological processes which can contribute to development of HF [6]. For example, miR-146b has been identified to play a protective role in cardiac ischemic injury [7]. miR-181-a is reported to regulate proliferation and apoptosis in human fibroblasts [8].

miR-200 has recently have been studied extensively and has been a hot topic of study. The family includes miR-200a. miR-200a is considered to be an important member, which has attracted more attention, as seen in studies. In a previous study it was identified that miR-200a targets the RNF11 gene and could result in regulation of necroptosis in cardiac cells and in animal models [9]. miR-200a has been identified to be involved in a number of cancer conditions [10-12]. Interestingly, it has been found that miR-200a is a negative regulator of high mobility group box-1 (HMGB1) and is a potential target of miR-200a [13]. Earlier it was reported that HMGB1 is a potential biomarker in heart transplantation and cell death in heart failure [14]. Looking into the above reports, the aim of the study was to evaluate the link between miR$200 a$ and HMGB1 in heart failure induced rats.

\section{Material and methods}

\section{Target population}

For the study we included about 180 subjects visiting the Department of Cardiology, Shanxi Provincial People's Hospital, Taiyuan, China, who included about 100 subjects with chronic heart failure whereas 80 were healthy. All the subjects were informed about the study and written consent was obtained. The subjects with chronic heart failure were confirmed after evaluation by three cardiologists visiting the department. The cardiologists followed the guidelines laid down by the NIHCE; also the subjects were graded in accordance to the NYHA classification as Class 2 (40 subjects), Class 3 (29 subjects) or Class 4 (31 subjects). Based on the etiology of the disease the subjects with chronic heart failure were grouped under the groups showing coronary heart disease (46 subjects), hypertension (44 subjects) and subjects showing cardiomyopathy (10 subjects). The subjects showing no clinical signs or an abnormal echocardiogram were regarded as the control group. All the study protocols were approved by the ethical review board of Shanxi Provincial People's Hospital, Taiyuan, China. All the subjects were informed about the study and written consent was obtained.

\section{Serum levels of HMGB1 in chronic heart failure subjects}

Blood was collected from subjects with chronic heart failure and also from the control followed by centrifugation to separate the serum, which was stored at $-80^{\circ} \mathrm{C}$. ELISA assay was performed for measuring the HMGB1 in the serum samples using an ELISA kit (Thermo Fisher USA) following the supplied instructions. Briefly, about $50 \mu \mathrm{l}$ of serum and standard from the kit were mixed and plated on 96-well plates which were previously coated with HMGB1 antibody. Before HMGB1 labeling with peroxidase secondary antibody, the well plates were rinsed with working buffer supplied with the kit. The reaction was stopped with sulfuric acid $(100 \mu \mathrm{l})$ before measuring the absorbance with a microplate reader at $450 \mathrm{~nm}$. The mean absorbance was determined by subtracting the readings from the blank.

\section{Isolation of exosome}

For isolating the cells and their fragments, the isolated serum was centrifuged at 10000 rpm for $10 \mathrm{~min}$. The supernatant was collected, mixed with thrombin $(5 \mathrm{U} / \mathrm{ml})$ and incubated for $10 \mathrm{~min}$ at room temperature. The resultant had precipitation buffer added to precipitate the exosome. The resultant was again centrifuged for $25 \mathrm{~min}$ at $10000 \mathrm{rpm}$ and re-suspended in nuclease free water $(20 \mu \mathrm{l})$. The obtained resultant solution was used for isolation of miR.

\section{Isolation of RNA}

The RNA from exosome was isolated using an RNA isolation kit (Thermo Fisher USA), Briefly, lysis buffer $(100 \mu \mathrm{l})$ was added to the obtained exosome pellet and was mixed for $20 \mathrm{~s}$. The resultant mix was stored and settled for 5 min for lysis. About $50 \mu$ l of ethanol was added and mixed for $15 \mathrm{~s}$. The obtained mixture was channelized into a spin column then centrifuged at $10000 \mathrm{rpm}$ ( $2 \mathrm{~min}$ ) and then rinsed at least three times with $100 \mu$ of washing buffer. The RNA was isolated and obtained by eluting with buffer $(20 \mu \mathrm{l})$.

\section{Quantitative real-time-PCR (qRT-PCR)}

For performing RT-qPCR, total RNA was isolated from exosomes using Trizol reagent (Invitrogen, USA) following supplied instructions. The RT-qPCR was done using a Bio-Rad real-time PCR system. Briefly, about $5 \mu \mathrm{l}$ of total RNA was subjected to reverse transcription to cDNA using a cDNA synthesis kit (Thermo Fisher USA) using RT primers for miRs. The qRT-PCR was done using a SYBR green assay. The sequences of primers are presented in Table I.

\section{Animal model of chronic heart failure}

All the animal protocols prior to the study were approved by the animal ethical review board of 
Table I. Primer sequences

\begin{tabular}{|lcc|}
\hline Name of gene & \multicolumn{1}{c|}{ Forward } & Reverse \\
\hline HMGB1 (Rat) & TGC AGA TGA CAA GCA GCC TT & GCT GCA TCA GGC TTT CCT TT \\
\hline GAPDH (Rat) & CAA GGT CAT CCA TGA CAA CTTTG & GTC CAC CAC CCT GTT GCT GTAG \\
\hline miR-200a (Rat) & GCCACCTTTACGGCATCGACG & AACTGGAGTACCGCTGGCCA \\
\hline HMGB1 (Human) & AAG GAG AAC ATC CTG GCC TGTC & TCC CAG TTT CTT CGC AAC ATCA \\
\hline GAPDH (Human) & CAT GAG AAG TAT GAC AAC AGCCT & AGT CCT TCC ACG ATA CCA AAGT \\
\hline miR-200a (Human) & ACGGTACCTACCGTTCGTGAC & CCACCTGGAGACTCCGACTG \\
\hline
\end{tabular}

Department of Cardiology, Shanxi Provincial People's Hospital, Taiyuan China. The approval number was $\mathrm{SH} 1002 \mathrm{CH}$. For developing a rat model of chronic heart failure Sprague-Dawley rats (male) aged 8 to 10 weeks weighing 225 to 250 g were obtained from Shanxi Provincial People's Hospital, Taiyuan China. All the animals were housed under controlled conditions of temperature $\left(25^{\circ} \mathrm{C}\right)$ and humidity (40\%). The rats were exposed to a 12 hour dark/light cycle and free access to pellet diet and water ad libitum. For inducing coronary heart failure in animals, the coronary artery of rats was operated on for ligation of the left anterior descending (LAD) artery between the auricular appendix and conus arteriosus. The rats after surgery received treatment of penicillin $(4 \mathrm{mg}$ / $\mathrm{kg}$ ) for 3 days. The chronic heart failure rats were evaluated for ejection fraction of the left ventricle. The rats with ejection fraction less than $45 \%$ were confirmed to be successfully induced for chronic heart failure and were included in the study.

\section{miR-200a transfection (in vivo)}

miR-200a mimic and plasmid were procured from Sigma Aldrich USA. The LV-HMGB1 i.e. HMGB1 plasmid and negative control were developed in GenePharma Ltd China. After the development of the chronic heart failure model, the rats were anesthetized and were then placed on a table in supine position and then injected with plasmid $(2 \mu \mathrm{l})$ into the tail vein. Twenty-eight days after transfection, an electrocardiogram was recorded and other cardiac parameters were studied.

\section{Evaluation of cardiac function}

The animals were subjected to pentobarbital anesthesia (2\%) and were fastened in a supine state. An echocardiogram (ECG) was taken to evaluate the left ventricular posterior wall dimension (LVPWD), left ventricular end diastolic dimension (LVEDD), left ventricular end systolic diameter (LVESD) and interventricular septal dimension (IVSD). The fractional shortening (FS) and LVEF were studied by ultrasonography.

\section{Hemodynamic parameter study}

After ECG studies, a catheter was introduced in the left ventricle via the right carotid artery. After this the left ventricular end diastolic pressure (LVEDP), left ventricular systolic pressure (LVSP), heart rate $(H R)$, ventricular pressure drop rate $(-\mathrm{d} p / \mathrm{d} t)$ and ventricular pressure rise rate $(+\mathrm{d} p /$ $\mathrm{d} t$ ) were recorded by a physiological recorder.

\section{Ratio of heart tissue weight to total body weight}

For this experiment the heart was isolated and was stored in normal saline solution for removing the blood from it. The heart was dissected into the left ventricle with septum, atria and right ventricle and all these parts were weighed. The right ventricular mass index (RVMI) and left ventricular mass index (LVMI) were compared to the complete body weight.

\section{Study of oxidative stress and mediators of inflammation in cardiac tissues}

Oxidative stress was studied in both tissue as well as blood samples. After evaluating the heart tissue weight studies, the heart was homogenized in tissue homogenizer and centrifuged at $10000 \mathrm{rpm}$ for $10 \mathrm{~min}$. The supernatant was processed for analysis of oxidative stress. For determining oxidative stress, activity of the antioxidant enzyme SOD was measured; also levels of malondialdehyde (MDA) were assessed by the ELISA method using a kit following the manufacturer's protocol. In addition to this expression of interleukin-1 (IL-1) and tumor necrosis factor (TNF- $\alpha$ ) was identified by the ELISA method following the supplier's instructions.

The blood was processed for removing serum by centrifugation. Serum was stored at $-80^{\circ} \mathrm{C}$. The levels of creatine kinase-MB, B-type natriuretic peptide, TNF- $\alpha$, IL-1, SOD and MDA were evaluated in plasma samples using an ELISA kit following the supplied instructions. 


\section{Western blot analysis}

For studying the expression of proteins, western blot analysis was performed. The proteins were separated from the heart tissues and homogenized in a tissue homogenizer. The tissue lysate was centrifuged at $10000 \mathrm{rpm}$ for $10 \mathrm{~min}$. The supernatants were further used for experiments. The total protein was determined using a protein estimation kit (Sigma Aldrich USA). About $20 \mu \mathrm{g}$ of protein was submitted to SDS-PAGE electrophoresis by loading it on $10 \%$ sodium dodecyl sulfate polyacrylamide gel and then transferred to PVDF membranes. The membranes were rinsed three times using TTBS for $5 \mathrm{~min}$ and were then incubated along with HRP-conjugated IIry antibody for $60 \mathrm{~min}$. The expression of proteins was studied by western blot analysis, GAPDH was selected as loading control.

\section{Luciferase activity assay}

The CDNA sequences of the WT-3'-UTR region of HMGB1 and MUT-3'-UTR of HMGB1 were combined and instilled in the $p$-miR GLO reported plasmid. The MUT-3'-UTR or WT-3'-UTR and miR-200a mimics or the negative control (NC) were transfected in the 293T cells with the help of Lipofectamine 3000 transfection reagent. The luciferase activity was evaluated using a dual luciferase reporter assay system.

A

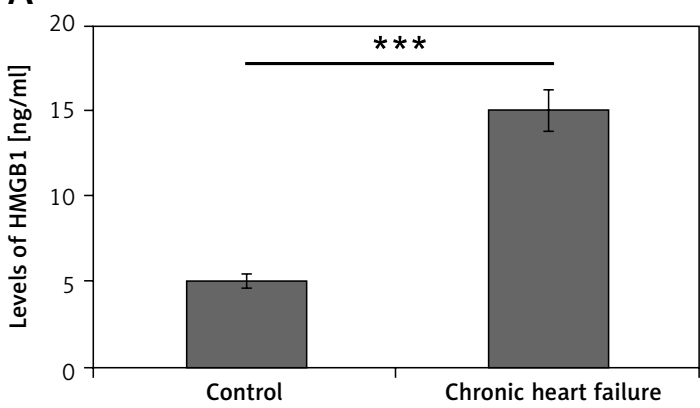

C

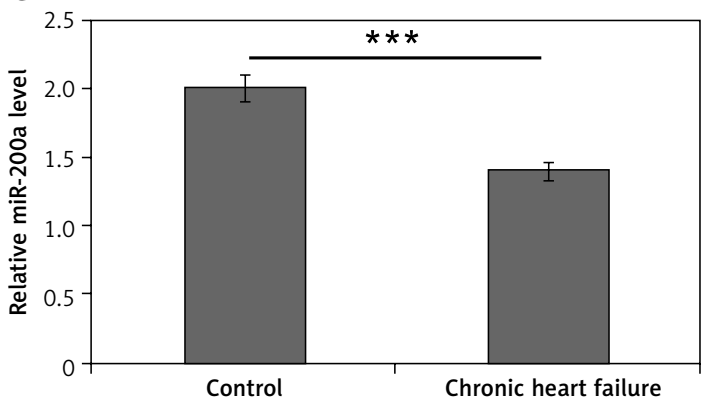

\section{Statistical analysis}

All the results were presented as mean \pm SD. The evaluation was performed using one-way analysis of variance (ANOVA) or Student's $t$ test. For studying the relation between miR-200a and HMGB1 in chronic heart failure subjects, Spearman's rank analysis was done. $P<0.05$ was defined to be significant.

\section{Results}

Levels of HMGB1 and miR-200a in serum of chronic heart failure and healthy subjects

For studying the levels of HMGB1 and miR200a in patients with chronic heart failure and normal subjects, a total of 180 subjects were included and blood samples were collected. About 100 samples were from subjects who reported with chronic heart failure and 80 were normal healthy control subjects. The ELISA method was employed for evaluating the serum levels of HMGB1 protein whereas the levels of miR-200a and mRNA HMGB1 were studied in serum via qRT$P C R$ assay. The outcomes demonstrated that the levels mRNA and protein HMGB1 were markedly increased in patients with chronic heart failure compared to the normal subjects (Figures $1 \mathrm{~A}, \mathrm{~B}$ ). On the other hand, the levels of miR-200a in serum

B

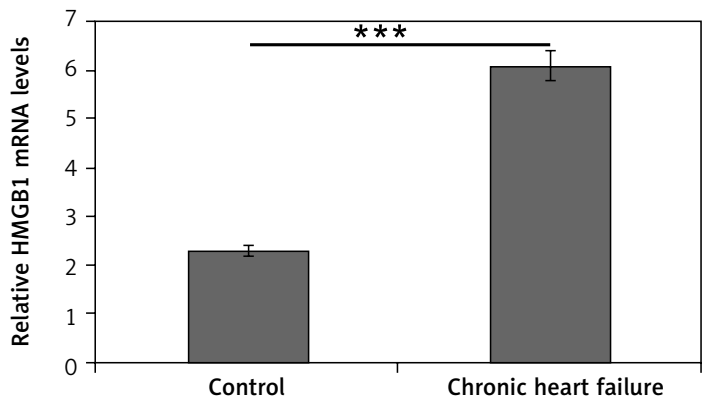

D

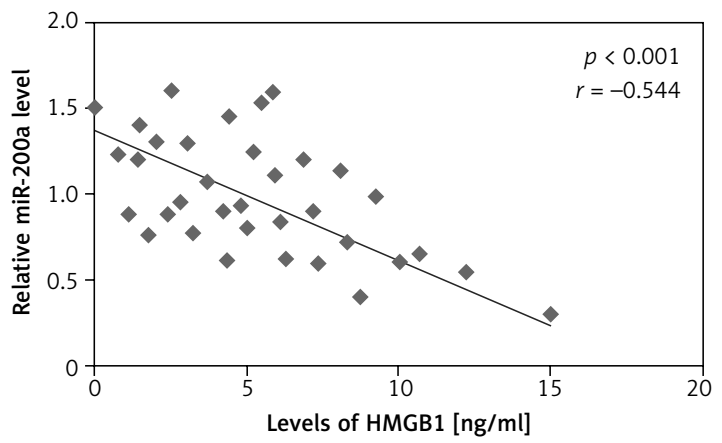

Figure 1. Serum profile of levels of HMGB1 and miR-200a in subjects reported for chronic heart failure. A - Results of ELISA analysis for serum levels of HMGB1 protein in chronic heart failure patients. B - qRT-PCR analysis for serum mRNA levels of HMGB1 in chronic heart failure subjects. C - qRT-PCR analysis of miR-200a levels in chronic heart failure subjects. D - Quantitative analysis showing inverse relation between levels of miR-200a and HMGB1 in chronic heart failure subjects evaluated by Spearman's rank correlation. The results are mean \pm SE. ${ }^{* * *} P<0.001$ compared to control 
were suppressed in subjects with chronic heart failure versus the control group (Figure $1 \mathrm{C}$ ). Interestingly, an inverse correlation was seen between the levels of HMGB1 and miR-200a in the serum of 100 chronic heart failure subjects (Figure $1 \mathrm{D}$ ).

\section{miR-200a increases LVSP, $+\mathrm{d} p / \mathrm{d} t,-\mathrm{d} p / \mathrm{d} t$ and decreases LVEDP and heart rate}

To evaluate the effect of miR-200a on cardiac activity in chronic heart failure rats we transfected the rats with miR-200a or the scrambled sequence (negative control). The hemodynamic parameters were evaluated. The outcomes showed no significant changes in the hemodynamic parameters of sham, normal, chronic heart failure and NC groups (Figure 2). As anticipated, the chronic heart failure rats showed higher heart rates and LVEDP but suppressed levels of

A

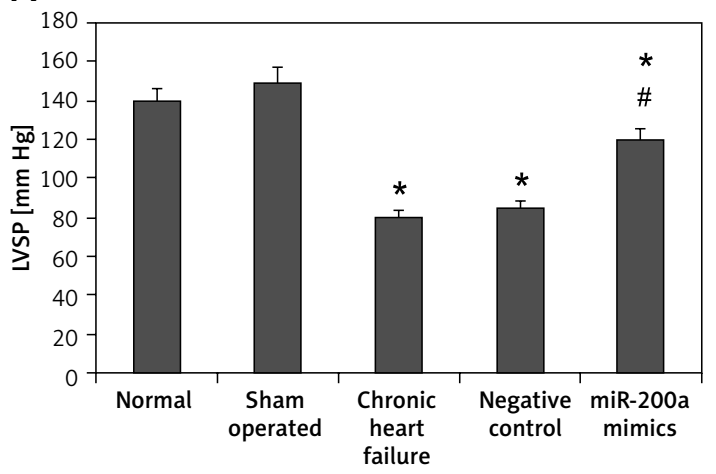

C

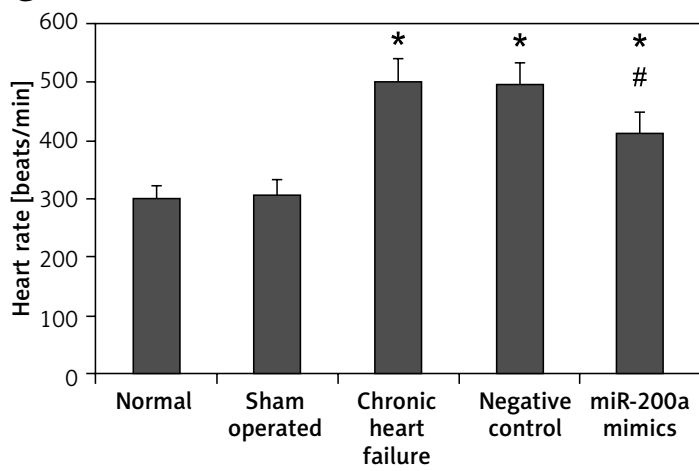

$E$

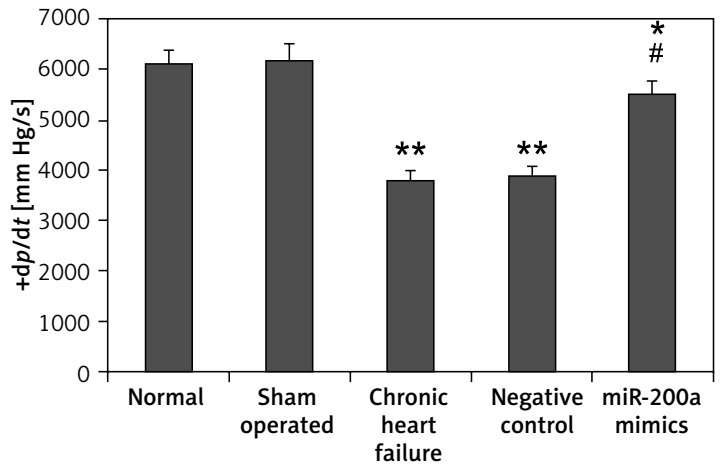

$+\mathrm{d} p / \mathrm{d} t$, - $\mathrm{d} p / \mathrm{d} t$, LVSP compared to sham operated rats (Figures $2 \mathrm{~A}-\mathrm{E}$ ). However, when compared to chronic heart failure rats, the miR-200a overexpressed rats showed significantly suppressed levels of LVEDP and heart rate, and high levels of $+\mathrm{d} p / \mathrm{d} t,-\mathrm{d} p / \mathrm{d} t$, LVSP (Figures 2 A-E).

\section{miR-200a decreased LVESD, LVPWD, LVEDD,} IVSD and increased FS and LVEF

The cardiac parameters such as LVESD, LVPWD, LVEDD, IVSD, FS and LVEF were recorded in rats by performing ultrasonography. It was observed that the chronic heart failure rats showed significantly increased levels of LVESD, LVPWD, LVEDD and IVSD whereas they demonstrated decreased levels of FS and LVEF (Figures $3 \mathrm{~A}-\mathrm{F}$ ). The results were interesting in chronic heart failure rats transfected with miR-200a, which showed decreased LVESD,

\section{B}

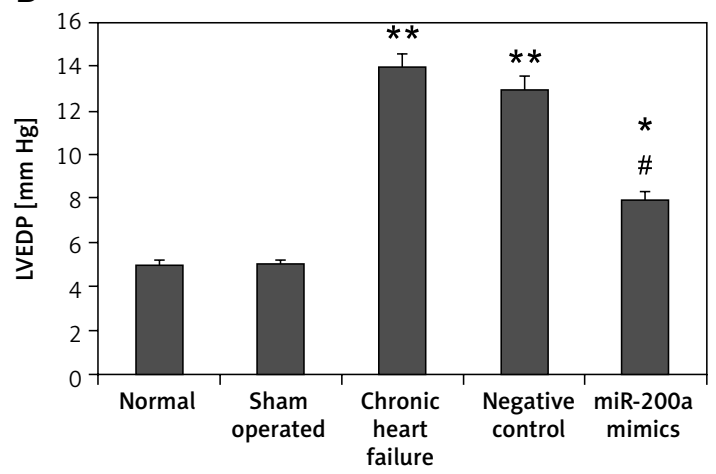

D

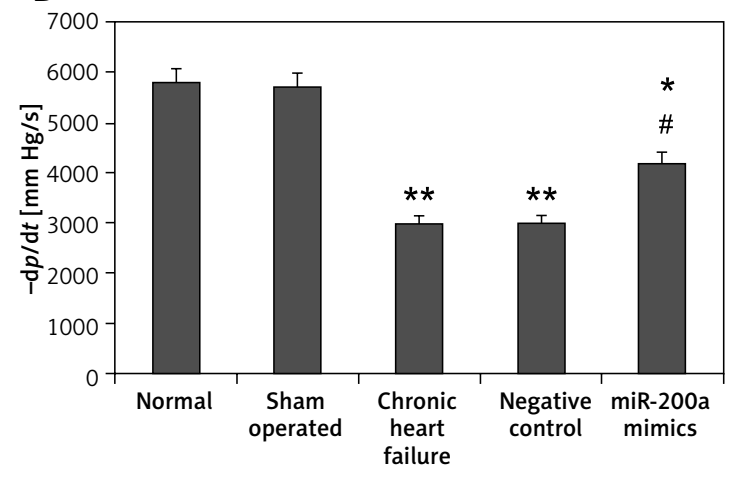

Figure 2. miR-200a attenuated the hemodynamic parameters in chronic heart failure rats. For the experiment the chronic heart failure rats were transfected with miR-200a mimics or negative control for 28 days. A - Evaluation of LVSP. B - Evaluation of LVEDP. C - Heart rate. D $--\mathrm{d} p / \mathrm{d} t$. E $-+\mathrm{d} p / \mathrm{d} t$. All the parameters were compared among five treatment groups of animals. All the results are presented as mean \pm SE. ${ }^{*} P<0.05,{ }^{* *} P<0.01$ compared to normal control. $\# P<0.05$ compared to chronic heart failure rats 
LVPWD, LVEDD, IVSD and increased FS and LVEF compared to negative control rats (Figures $3 \mathrm{~A}-\mathrm{F}$ ). However, there was no notable difference in the cardiac features of sham, normal, miR-NC and chronic heart failure rats (Figures $3 \mathrm{~A}-\mathrm{F}$ ).

\section{miR-200a suppressed the levels of RVMI and LVMI}

To evaluate the effect of miR-200a on heart function in chronic heart failure animals, we evaluated the ventricular mass index. The results showed that both RVMI and LVMI were increased remarkably in chronic heart failure rats compared to the sham operated and control rats. It was also observed that compared to chronic heart failure

A

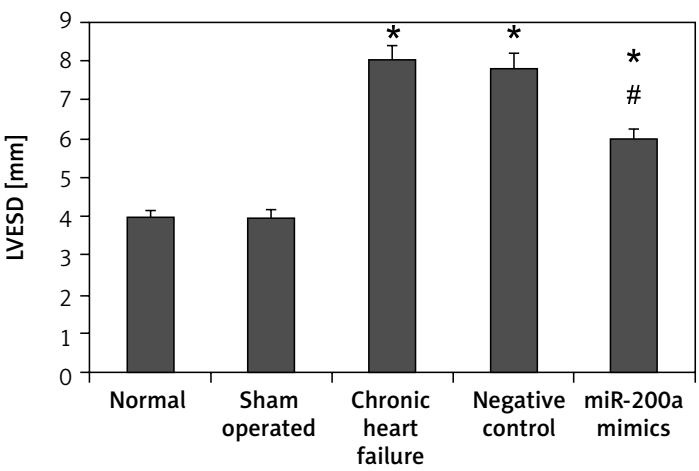

C

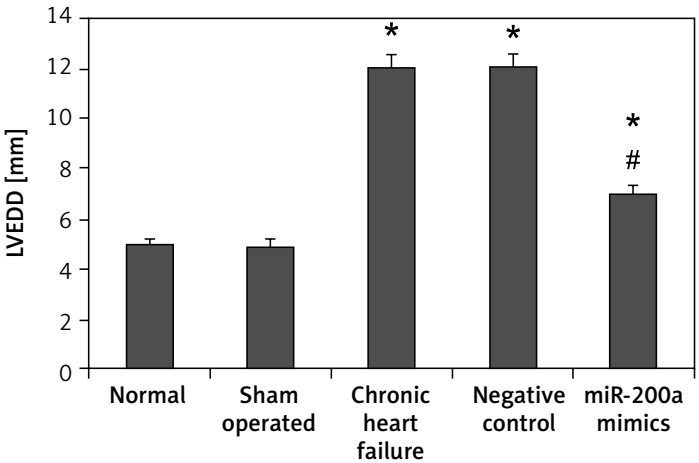

E

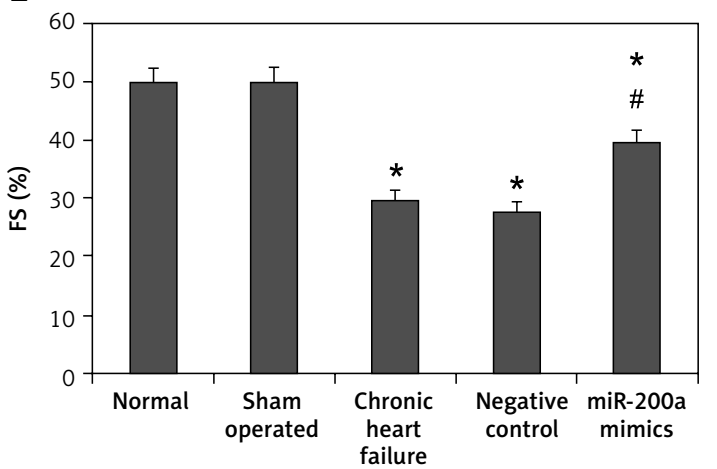

rats the miR-200a transfected rats demonstrated significantly decreased levels of RVMI and LVMI (Figures 4 A, B).

miR-200a decreases the levels of IL-1, TNF- $\alpha$, BNP, MDA, CK-MB and increases the activity of SOD in cardiac cells and serum of chronic heart failure rats

We evaluated the effect of miR-200a on the myocardial infarction markers BNP and CK-MB, markers of oxidative stress SOD and MDA, and inflammatory mediators IL- 1 and TNF- $\alpha$ in chronic heart failure rats (Figures $5 \mathrm{~A}-\mathrm{F}$ ). Likewise, the overexpression of miR-200a in chronic heart failure rats caused significantly decreased serum lev-

B

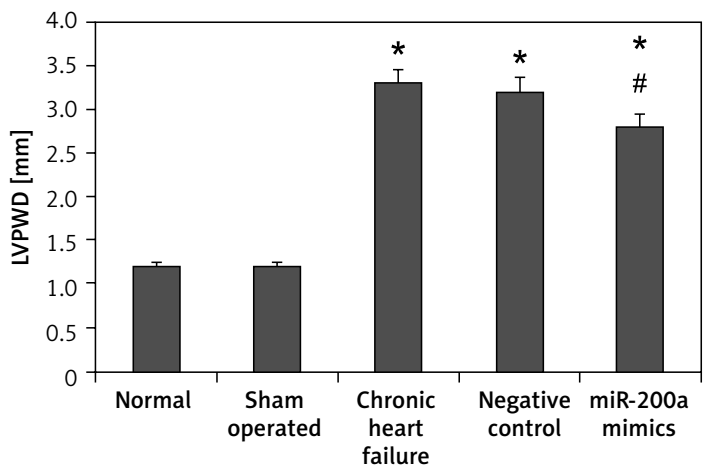

D

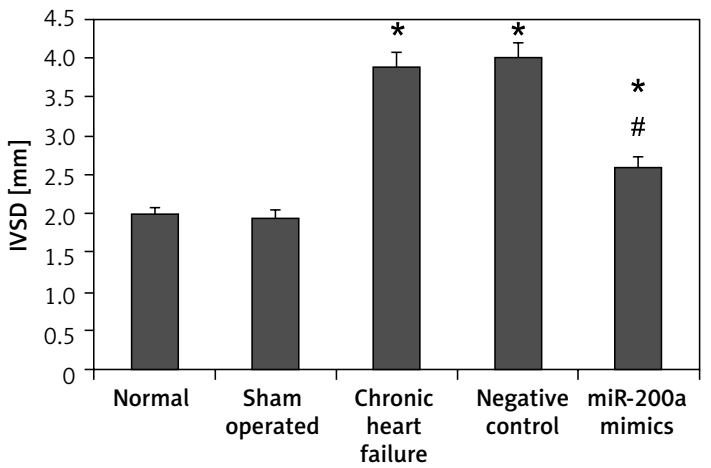

$\mathrm{F}$

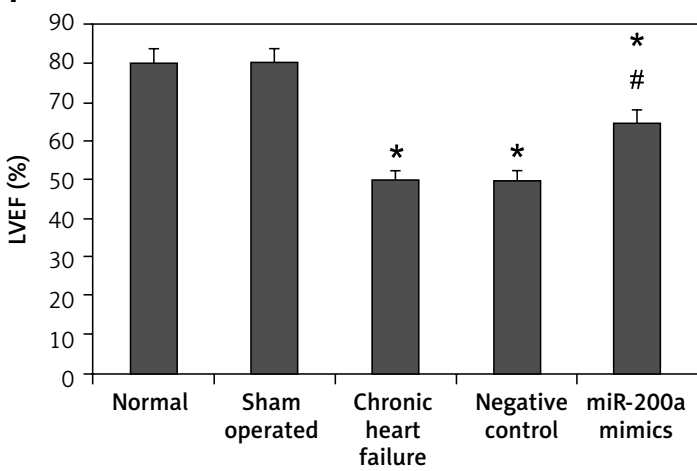

Figure 3. miR-200a attenuated the cardiac function in chronic heart failure rats. The chronic heart failure rats were transfected with miR-200a mimics or negative control for 28 days. The animals were evaluated by electrocardiogram for: LVESD (A), LVPWD (B), LVEDD (C), IVSD (D), FS (E), LVEF (F). The results were compared among five groups with defined treatments. The results are mean \pm SE. ${ }^{*} P<0.05$ compared to normal rats. $\# P<0.05$ compared to chronic heart failure rats 
A

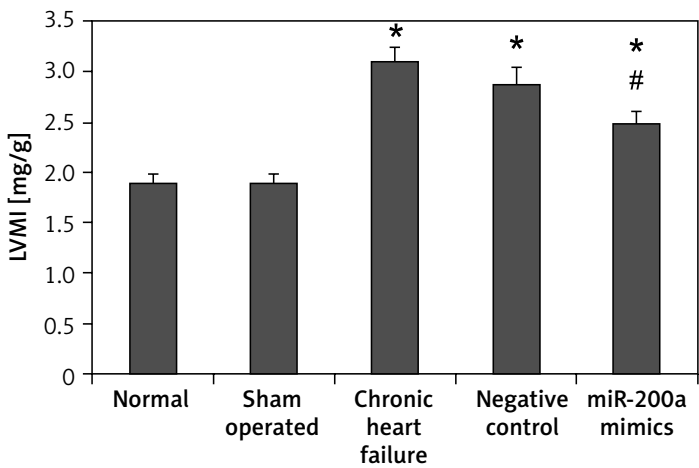

B

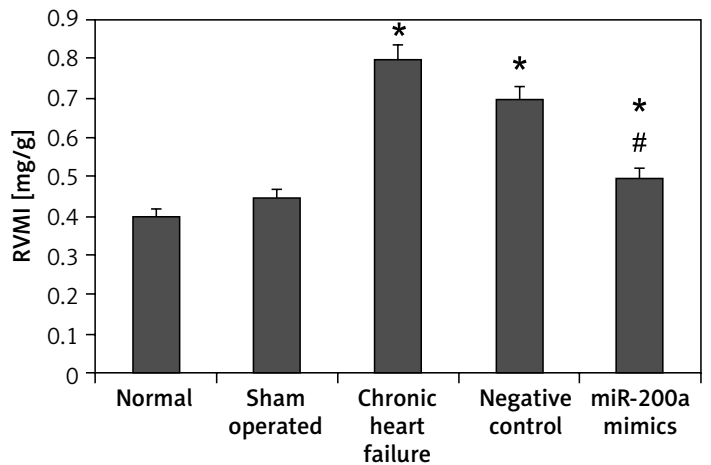

Figure 4. miR-200a enhanced the left and right ventricular mass index in chronic heart failure rats. A - LVMI, B - RVMI. The chronic heart failure rats were transfected with miR-200a mimics or negative control for 28 days. Both left and right ventricles were weighed and their ratio to body weight was compared among the treated groups. The data are presented as mean \pm SE. ${ }^{*} P<0.05$ compared to normal group. ${ }^{*} P<0.05$ compared to chronic heart failure rats

els of IL-1, TNF- $\alpha$, BNP, MDA, CK-MB and increased levels of SOD (Figures $5 \mathrm{~A}-\mathrm{F}$ ).

Further in our study we evaluated the effect of miR-200a on oxidative stress and mediators of inflammation in cardiac cells. The outcomes were parallel to those of serum levels; it was observed that the levels of MDA (oxidative stress marker) along with IL-1, MDA and TNF- $\alpha$ (inflammatory mediators) were markedly increased whereas the activity of SOD decreased in cardiac cells of rats with chronic heart failure compared to sham operated and control rats (Figures 6 A-D). However, the cells isolated from miR-200a transfected rats showing upregulated miR-200a demonstrated a decrease in levels of MDA, IL-1, TNF- $\alpha$ and increased SOD activity compared to cardiac cells of chronic heart failure rats (Figures 6 A-D).

miR-200a decreased expression of HMGB1 in cardiac tissues of chronic heart failure rats

The results of qRT-PCR and western blot analysis suggested that the levels of miR-200a were suppressed whereas the protein and mRNA levels of HMGB1 were increased in the myocardial tissues of chronic heart failure rats compared to the sham and control group (Figures 7 A-D). The levels of miR-200a were significantly elevated in the miR-200a group rats compared to the rest of the rats, suggesting the transfection of miR$200 a$ in rats. It was also found that the transfection of miR-200a caused a reduction in both protein and mRNA levels of HMGB1 in myocardial tissues.

\section{HMGB1 is a potential target of miR-200a}

Previously it has been reported that HMGB1 is a potential target of miR-200a by directly binding to the 3'UTR region, which complements the seed region of miR-200a [13] (Figure $8 \mathrm{~A}$ ). In our study, the outcome of luciferase assay suggested that overexpression of miR-200a caused a significant decrease in luciferase activity of wild type and not in the CDNA sequence of mutant and HMGB1 (Figure $8 \mathrm{~B}$ ). To further confirm the involvement of HMGB1 in cardiac activity influenced by miR200a, the chronic heart failure rats were transduced with miR-200a or with miR-200a along with HMGB1 overexpressing plasmid. It was observed that the protein as well as mRNA levels of HMGB1 were elevated in the cardiac tissues of chronic heart failure HMGB1 transfected rats transfected along with miR-200a versus the rats treated with only miR-200a (Figures 8 C-E). The results of ELISA analysis suggested that as compared to the heart failure rats with miR-200a transfection the rats transfected with HMGB1 and miR-200a demonstrated increased levels of IL-1, MDA, TNF- $\alpha$ and decreased levels of SOD (Figures $8 \mathrm{~F}, \mathrm{G}$ ).

\section{Discussion}

Recently a number of studies about miR based therapies have emerged. To date, numerous miRs have been discovered and have been proved to be involved in various cardiovascular pathologies. In the case of heart failure and other cardiac pathologies, miRs have been reported to play a crucial role $[5,6]$. Studies have shown great potential of miRs for their diagnostic as well as therapeutic approaches in heart failure $[15,16]$.

Studies about circulating proteins as potential markers for diagnostic purposes in heart failure have increased our knowledge about this abnormality. The markers includes cardiac specific proteins such as LOX-1, the interleukin class of proteins, TNF- $\alpha$ and brain natriuretic peptide (BNP) $[17,18]$. With the involvement of advanced tech- 
A

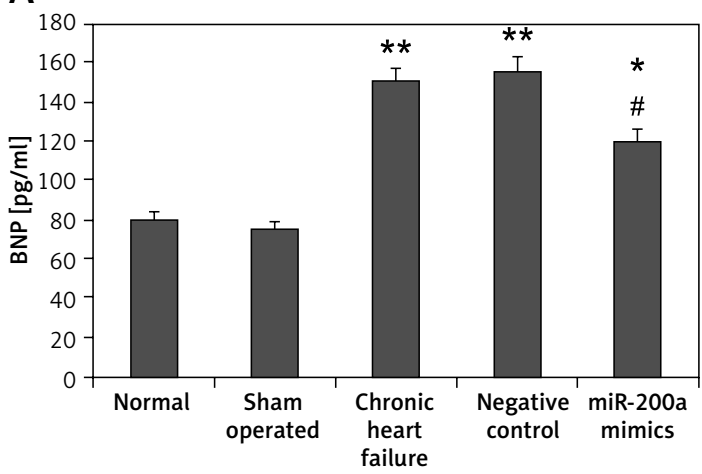

C

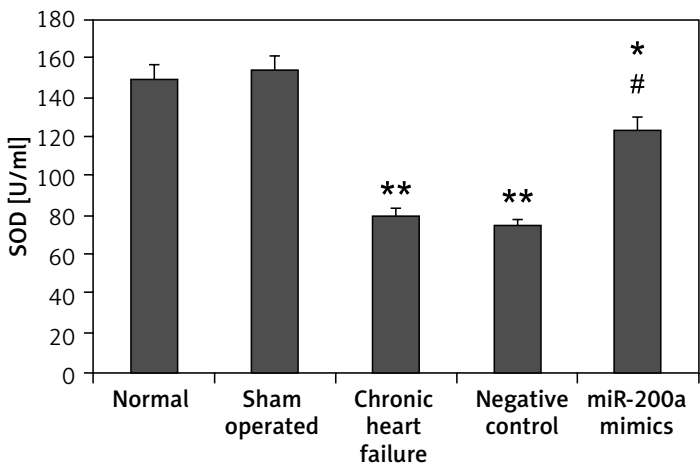

E

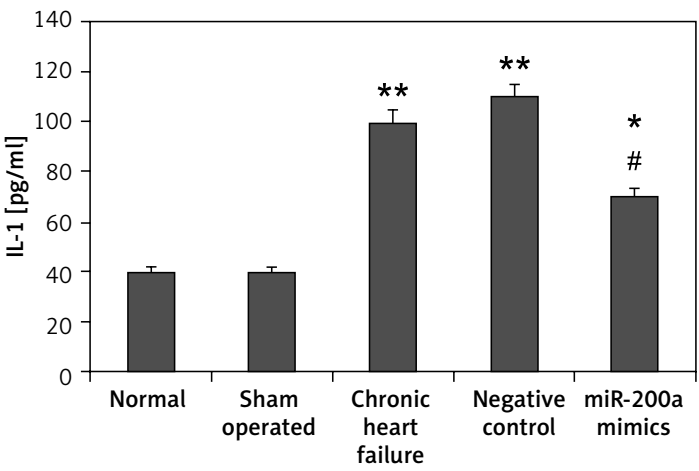

B

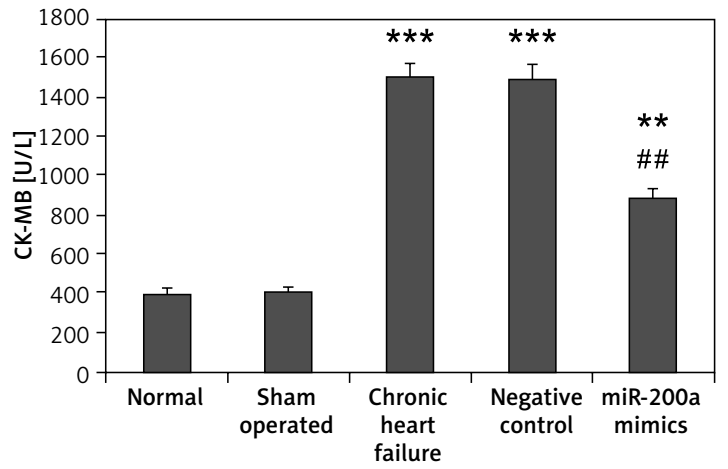

D

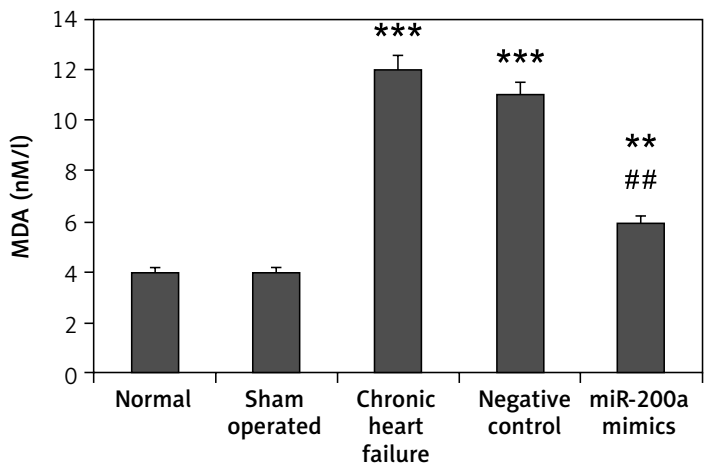

$\mathrm{F}$

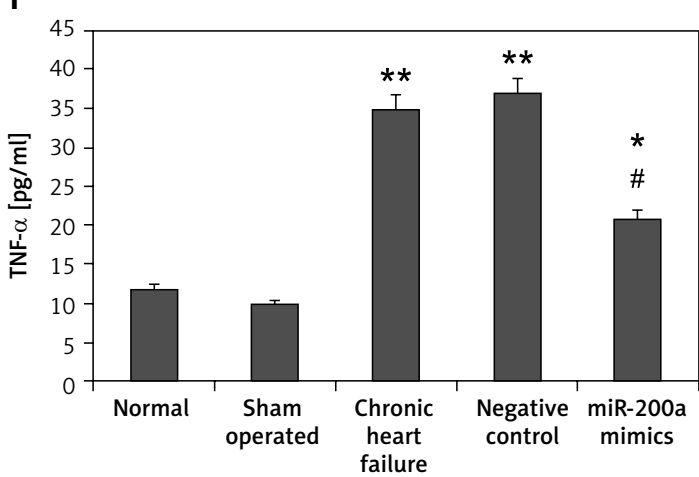

Figure 5. miR-200a improves the status of inflammatory factors and oxidative stress in chronic heart failure rats. The chronic heart failure rats were transfected with miR-200a mimics or negative control for 28 days after which the serum levels of: BNP (A), CK-MB (B), SOD (C), MDA (D), IL-1 (E), and TNF- $\alpha(\mathbf{F})$ were evaluated by ELISA. The results are mean \pm SE. ${ }^{*} P<0.05,{ }^{* *} p<0.01$, and ${ }^{* * *} p<0.001$ compared with normal rats. ${ }^{\#} P<0.05$ and ${ }^{\# \#} p<0.01$ compared to chronic heart failure rats

niques such as qRT-PCR and microarray profiling the possibilities of employing miRs for the diagnostic as well as therapeutic approach in heart failure have increased. Recently these techniques have been employed in successfully identifying miRs such as miR-125a-5p, miR-190a, miR-638, miR-550, miR-211-5p, miR-200a, miR-545-5p and miR-494 in patients with heart failure. Among these miRs a few were identified to be involved in regulation of essential genes responsible for remodeling of the heart [19]. Interestingly, previous reports have established that miR-200a exerts protective action in cardiomyocytes against hypoxia-induced apoptosis via suppressing Keap1 [20].
HMGB1, also known as High mobility group box 1 protein, is among one of the major proteins found in eukaryotes. The biological potential of this protein is dependent on its location in cells. HMGB1 in the nucleus is involved in many important processes of DNA such as transcription, stability of genes and repair of DNA, and in the cytoplasm it is involved in regulation of autophagy. HMGB1 is identified to play an important role in processes such as proliferation, migration, differentiation, inflammation and regeneration of tissues. Due to its involvement in a number of important processes, HMGB1 has been studied in detail in cardiac abnormalities [21]. Earlier reports have emerged 
A

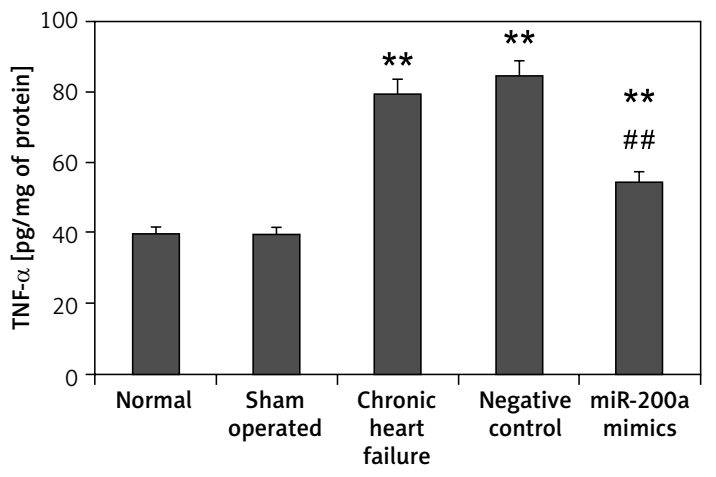

C

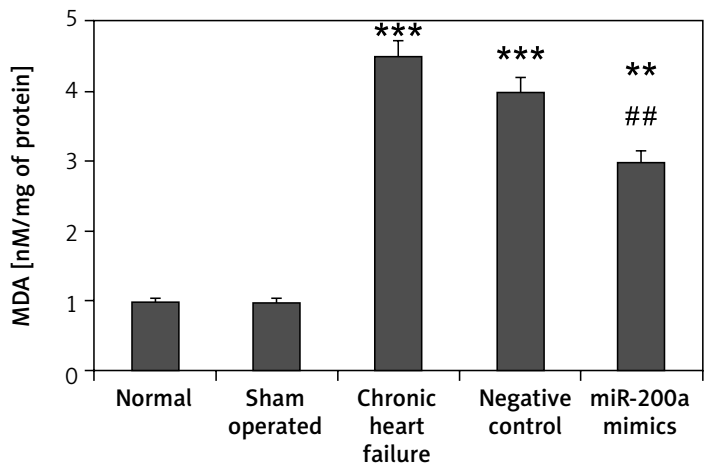

B

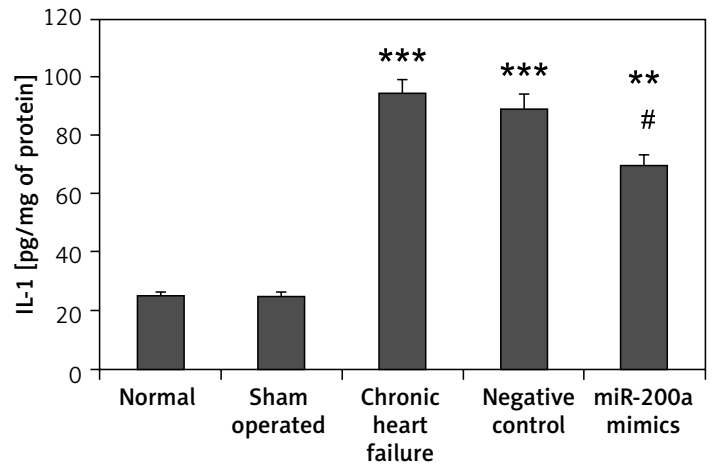

D

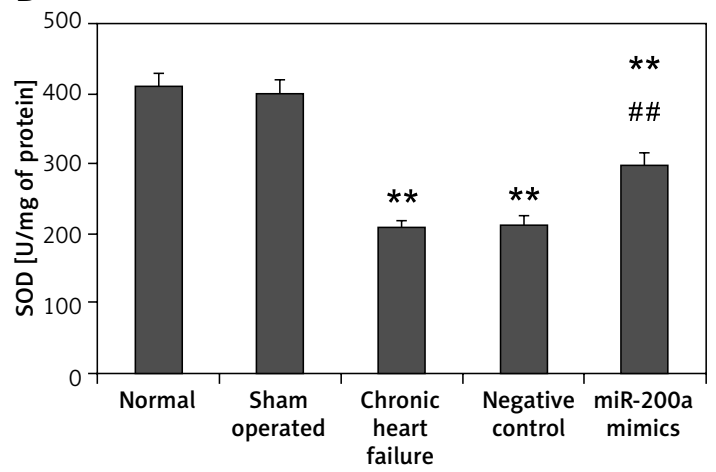

Figure 6. miR-200a improved the status of oxidative stress and inflammatory factors in the cardiac tissues of chronic heart failure rats. The chronic heart failure rats were transfected with miR-200a mimics or negative control for 28 days; the levels of inflammatory mediators: TNF- $\alpha$ (A), IL-1 (B) and oxidative stress markers: MDA (C), and SOD (D) were evaluated by ELISA. Data are presented as mean \pm SD. The results are mean \pm SE. ${ }^{\star} P<0.05$, ${ }^{* *} p<$ 0.01 , and ${ }^{\star \star *} p<0.001$ compared with normal rats. ${ }^{\#} P<0.05$ and ${ }^{\# \#} p<0.01$ compared to chronic heart failure rats

A

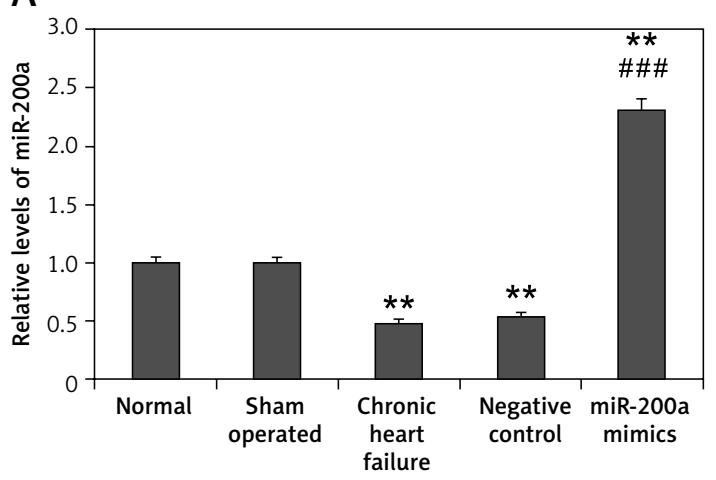

C
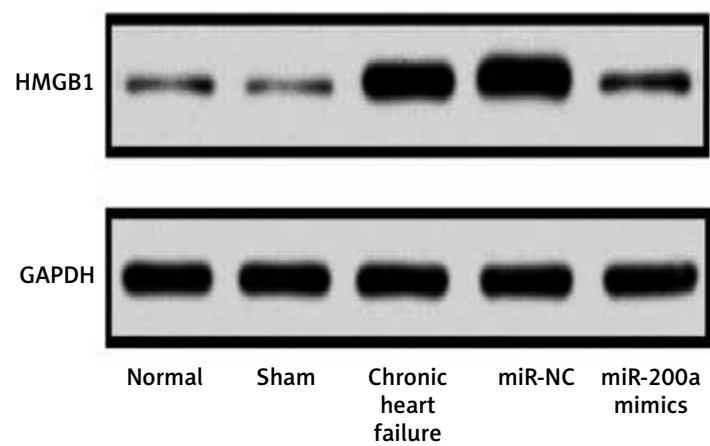

B

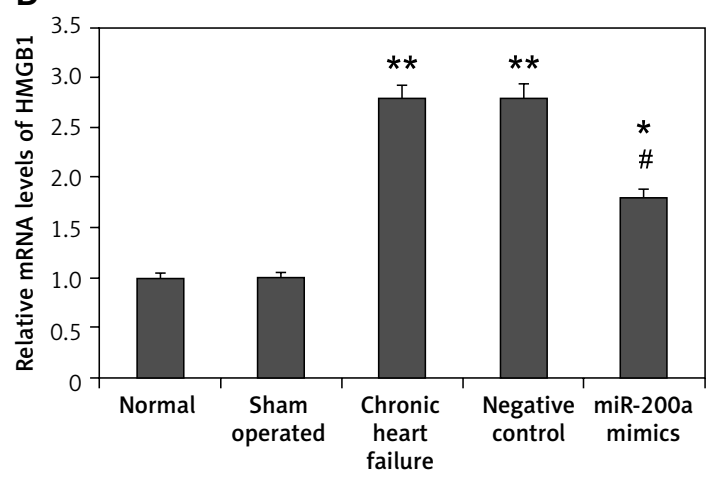

D

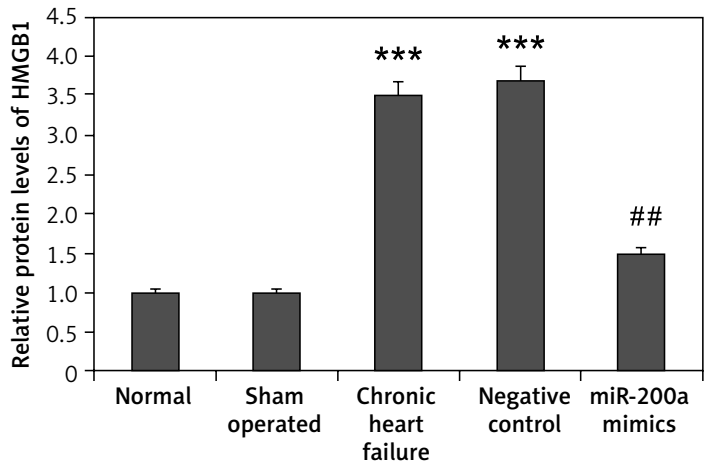

Figure 7. miR-200a suppressed the protein as well as mRNA levels of HMGB1 in cardiac tissues of chronic heart failure rats. The chronic heart failure rats were transfected with miR-200a mimics or negative control for 28 days and qRT-PCR was performed for the analysis of: miR-200a (A) and HMGB1 mRNA (B) levels in the cardiac tissues. C - The protein levels of HMGB1 in the cardiac tissues were assessed by western blot analysis. D - Quantitative analysis results $f$ or protein levels of HMGB1. The results are mean \pm SE. ${ }^{*} P<0.05,{ }^{* *} p<0.01$, and ${ }^{* * *} p<0.001$ compared with normal rats. ${ }^{\#} P<0.05,{ }^{\# \#} p<0.01$ and ${ }^{\# \#} p<0.001$ compared to chronic heart failure rats 
A

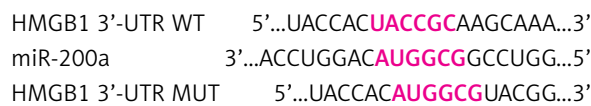

C

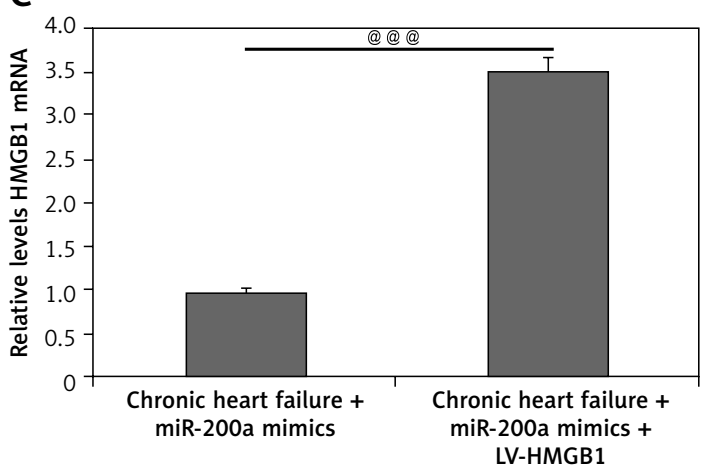

E

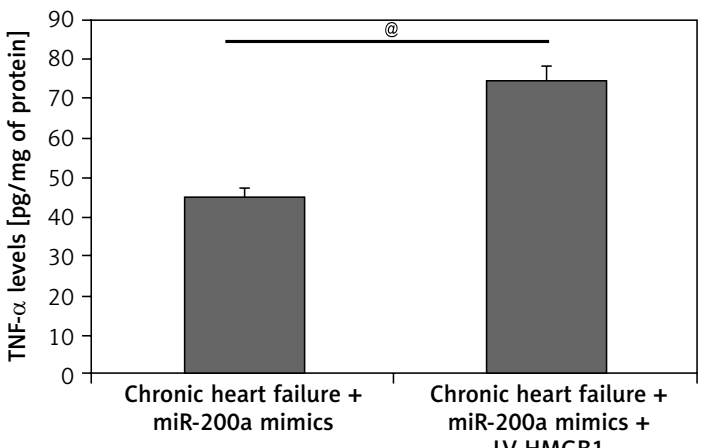

G

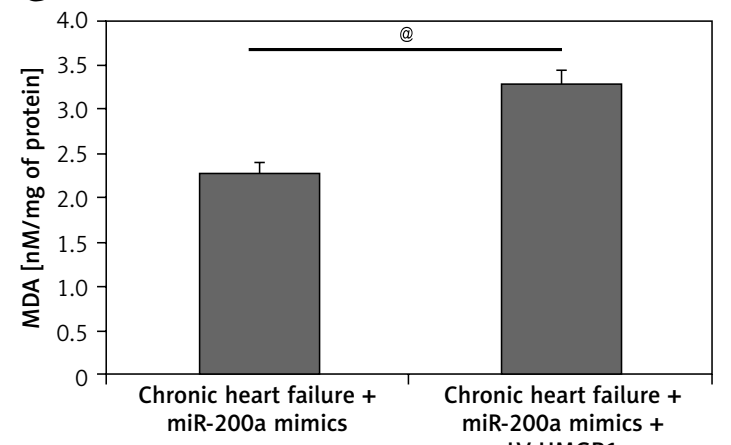

B
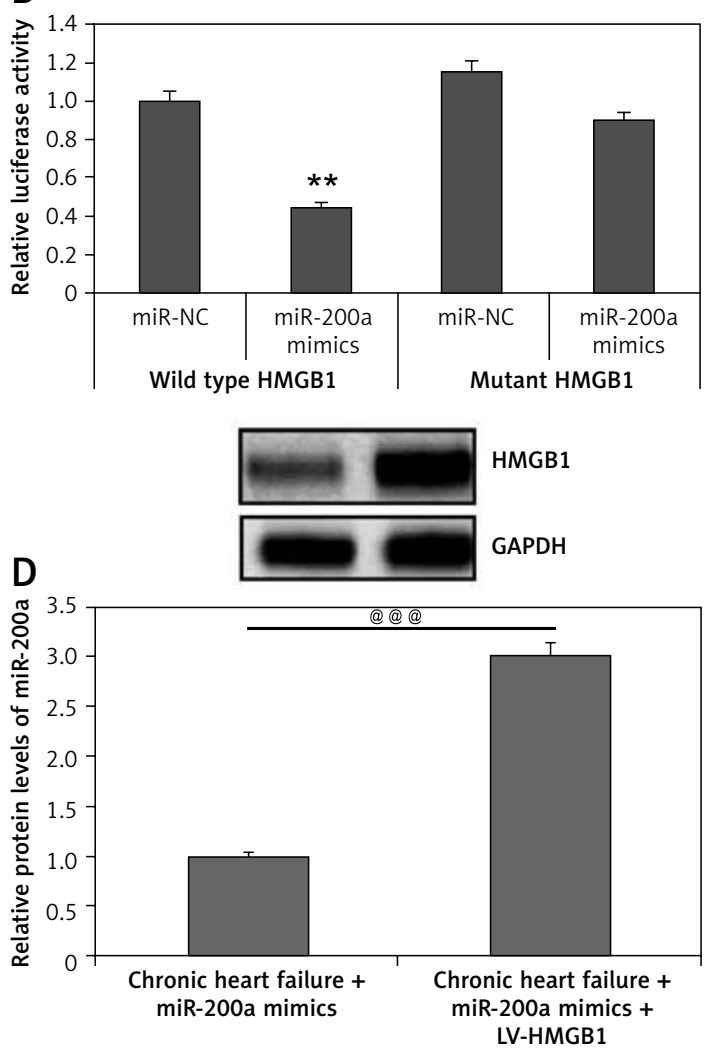

$\mathrm{F}$

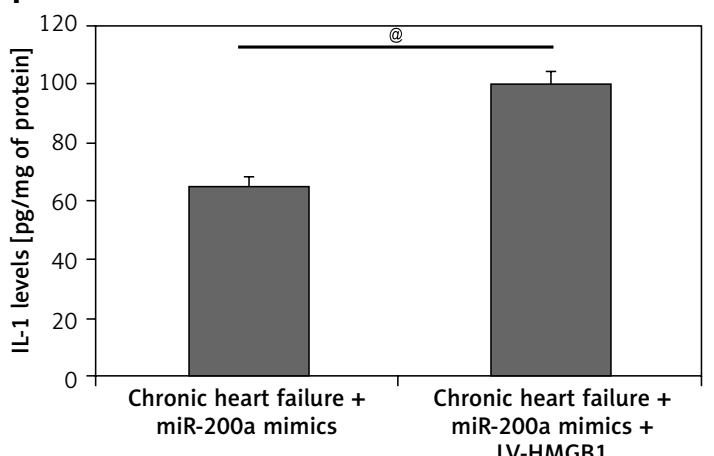

$\mathrm{H}$

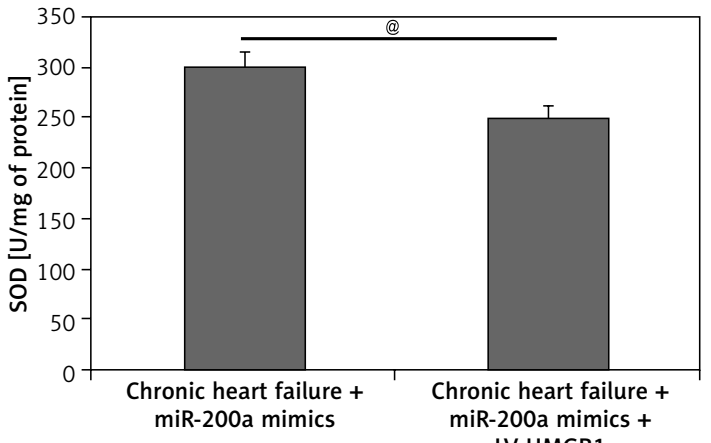

LV-HMGB1

Figure 8. miR-200a improved the status of oxidative stress and mediators of inflammation in rats with chronic heart failure via targeting HMGB1. A - Binding sequences showing potential binding sites of miR-200a on the 3'-UTR region of HMGB1 mRNA and the mutant. B - The wild type HMGB1 or the mutant HMGB1 was co-transfected with miR-200a mimics or respective negative control in the 293T cells. The luciferase activity was measured after 48 h. C - Relative HMGB1 mRNA levels were evaluated by qRT-PCR in chronic heart failure rats transfected with miR-200 mimics or miR-200a mimics + LV-HMGB1 for 28 days. D - Western blot analysis was done to evaluate the protein levels of HMGB1 in cardiac tissues. Quantitative results for protein levels of miR-200a. E-H - Results of ELISA for levels of TNF- $\alpha$, IL-1, MDA and SOD in cardiac tissues. The results are mean $\pm \mathrm{SE}$. ${ }^{*} P<0.01$ in comparison with miR-NC group. ${ }^{\circledR} P<0.05$ and ${ }^{\circledR ®} P<0.001$ compared to defined groups 
which confirmed upregulation of HMGB1 in subjects with myocardial infarction and coronary heart disease $[22,23]$. Here we evidenced that the HMGB1 serum levels and miR-200a were upregulated and downregulated respectively in subjects with heart failure compared to normal controls, and may hence act as biomarkers in diagnosis of heart failure.

miR-200a is reported to inhibit epithelial mesenchymal transition of pancreatic cancer and interestingly HMGB1 is found to be a potential target of miR-200a [13, 24]. Earlier it was evidenced that the proliferation of hepatic carcinoma is dependent on miR-200a via the HMGB1/RAGE axis [13]. However, the involvement of miR-200a and its target gene HMGB1 in heart failure remains unexplored.

In the present work, we established a chronic heart failure model and found that upregulation of miR-200a in chronic heart failure rats caused downregulation in levels of IL-1, TNF- $\alpha$ and MDA, whereas it caused upregulation in activity of SOD both in cardiac cells and serum of chronic heart failure rats. Oxidative stress is a remarkable feature of chronic cardiac abnormalities. Cell apoptosis and inflammation are the end results of oxidative stress causing fibrosis, cardiac injury and in extreme conditions heart failure [25-27]. A number of studies have suggested that miR-200a halts apoptosis in various cancer conditions [28, 29]. Also miR-200a has been reported to downregulate mediators of inflammation such as IL-1 and TNF- $\alpha$ and also suppress the levels of HMGB1 [13]. Our results suggested that transduction of miR-200a for upregulating the expression of miR-200a decreased oxidative stress and expression of mediators of inflammation in cardiac cells and exerted a protective effect on cardiac cells against injury in chronic heart failure rats [30].

Aiming to find the involved mechanism, we directed our focus on the levels of HMGB1. HMGB1 is a protein majorly discharged by dead cells [31]. HMGB1 is also reported to be an important marker for myocardial infarction [22]. Overexpression of HMGB1 causes apoptosis of cardiac cells and cardiac fibrosis [31]. Previous reports have confirmed HMGB1 as the direct target of miR-200a in hepatic cancer [13]. Here for the first time we report HMGB1 as a potential target of miR-200a in myocardial cells. We evidenced that miR-200a caused a significant decrease in expression levels of HMGB1 both in vitro and in vivo, and also it was noted that forceful expression of HMGB1 diminished the protective effect of miR-200a in cardiac cells as well as in chronic heart failure rats. Hence we demonstrated that miR-200a exerts protective action on the cardiac activity in chronic heart failure rats via the HMGB1 axis.
In conclusion, the present work showed that upregulation of miR-200a could decrease the levels of HMGB1 and also reduce inflammation and oxidative stress in cardiac cells. It is found that miR-200a leads to promotion and survival of cardiac cells by modulating the cardiac injury in chronic heart failure rats.

\section{Conflict of interest}

The authors declare no conflict of interest.

\section{References}

1. Inamdar AA, Inamdar AC. Heart failure: diagnosis, management and utilization. J Clin Med 2016; 5: E62.

2. Savarese G, Lund LH. Global public health burden of heart failure. Cardiac Fail Rev 2017; 3: 7-11.

3. Choi HM, Park MS, Youn JC. Update on heart failure management and future directions. Korean J Intern Med 2019; 34: 11-43.

4. Azad N, Lemay G. Management of chronic heart failure in the older population. J Geriatr Cardiol 2014; 11: 329-37.

5. Yan H, Ma F, Zhang Y, et al. miRNAs as biomarkers for diagnosis of heart failure: a systematic review and meta-analysis. Medicine (Baltimore) 2017; 96: e6825.

6. Murach KA, McCarthy JJ. MicroRNAs, heart failure, and aging: potential interactions with skeletal muscle. Heart Fail Rev 2017; 22: 209-18.

7. Di YF, Li DC, Shen YQ, et al. MiR-146b protects cardiomyocytes injury in myocardial ischemia/reperfusion by targeting Smad4. Am J Transl Res 2017; 9: 656-63.

8. Rang Z, Wang Z, Pang Q, Wang Y, Yang G, Cui F. MiR-181a targets PHLPP2 to augment AKT signaling and regulate proliferation and apoptosis in human keloid fibroblasts. Cell Physiol Biochem 2016; 40: 796-806.

9. Yang T, Cao C, Yang J, et al. miR-200a-5p regulates myocardial necroptosis induced by Se deficiency via targeting RNF11. Redox Biol 2018; 15: 159-69.

10. Wang X, Huang S, Li X, et al. A potential biomarker hsamiR-200a-5p distinguishing between benign thyroid tumors with papillary hyperplasia and papillary thyroid carcinoma. PLoS One 2018; 13: e0200290.

11. Xiao H, Tang K, Liu P, et al. LncRNA MALAT1 functions as a competing endogenous RNA to regulate ZEB2 expression by sponging miR-200s in clear cell kidney carcinoma. Oncotarget 2015; 6: 38005-15.

12. Gao C, Peng FH, Peng LK. MiR-200c sensitizes clear-cell renal cell carcinoma cells to sorafenib and imatinib by targeting heme oxygenase-1. Neoplasma 2014; 61: 680-9.

13. Li S, Huang Y, Huang Y, et al. The long non-coding RNA TP73-AS1 modulates HCC cell proliferation through miR-200a-dependent HMGB1/RAGE regulation. J Exp Clin Cancer Res 2017; 36: 51.

14. Kang R, Chen R, Zhang Q, et al. HMGB1 in health and disease. Mol Aspects Med 2014; 40: 1-116.

15. Bonneau E, Neveu B, Kostantin E, Tsongalis GJ, De Guire V. How close are miRNAs from clinical practice? A perspective on the diagnostic and therapeutic market. EJIFCC 2019; 30: 114-27.

16. Yang L, Wang B, Zhou Q, et al. MicroRNA-21 prevents excessive inflammation and cardiac dysfunction after myocardial infarction through targeting KBTBD7. Cell Death Dis 2018; 9: 769. 
17. Aspromonte N, Gulizia MM, Clerico A, et al. ANMCO/ ELAS/SIBioC Consensus Document: biomarkers in heart failure. Eur Heart J Suppl 2017; 19 (Suppl D): D102-12.

18. Welsh P, Kou L, Yu C, et al. Prognostic importance of emerging cardiac, inflammatory, and renal biomarkers in chronic heart failure patients with reduced ejection fraction and anaemia: RED-HF study. Eur J Heart Fail 2018; 20: 268-77.

19. Wong LL, Armugam A, Sepramaniam S, et al. Circulating microRNAs in heart failure with reduced and preserved left ventricular ejection fraction. Eur J Heart Fail 2015; 17: 393-404.

20. Sun X, Zuo H, Liu C, Yang Y. Overexpression of miR200 a protects cardiomyocytes against hypoxia-induced apoptosis by modulating the kelch-like ECH-associated protein 1-nuclear factor erythroid 2-related factor 2 signaling axis. Int J Mol Med 2016; 38: 1303-11.

21. Pellegrini L, Foglio E, Pontemezzo E, Germani A, Russo MA, Limana F. HMGB1 and repair: focus on the heart. Pharmacol Ther 2019; 196: 160-82.

22. Li W, Sama AE, Wang H. Role of HMGB1 in cardiovascular diseases. Curr Opin Pharmacol 2006; 6: 130-5.

23. Yao HC, Zhou M, Zhou YH, et al. Intravenous high mobility group box 1 upregulates the expression of HIF-1alpha in the myocardium via a protein kinase B-dependent pathway in rats following acute myocardial ischemia. Mol Med Rep 2016; 13: 1211-9.

24. Lu Y, Lu J, Li X, et al. MiR-200a inhibits epithelial-mesenchymal transition of pancreatic cancer stem cell. BMC Cancer 2014; 14: 85.

25. Elahi MM, Kong YX, Matata BM. Oxidative stress as a mediator of cardiovascular disease. Oxid Med Cell Longev 2009; 2: 259-69.

26. Ikwegbue PC, Masamba P, Oyinloye BE, Kappo AP. Roles of heat shock proteins in apoptosis, oxidative stress, human inflammatory diseases, and cancer. Pharmaceuticals 2017; 11: E2.

27. Mukhopadhyay P, Eid N, Abdelmegeed MA, Sen A. Interplay of oxidative stress, inflammation, and autophagy: their role in tissue injury of the heart, liver, and kidney. Oxid Med Cell Longev 2018; 2018: 2090813.

28. Kozak J, Wdowiak P, Maciejewski R, Torres A. Interactions between microRNA-200 family and Sestrin proteins in endometrial cancer cell lines and their significance to anoikis. Mol Cell Biochem 2019; 459: 21-34.

29. Li M, Li J, Ye C, Wu W, Cheng Y. miR-200a-3p predicts prognosis and inhibits bladder cancer cell proliferation by targeting STAT4. Arch Med Sci DOI: https://doi. org/10.5114/aoms.2019.89969.

30. Martinotti S, Patrone M, Ranzato E. Emerging roles for HMGB1 protein in immunity, inflammation, and cancer. Immunotargets Therapy 2015; 4: 101-9. 